\title{
A pathological complete response to neoadjuvant chemotherapy and immunotherapy in a non-small cell lung cancer patient
}

\author{
Pengqiang Gao^, Hao Chen, Taidui Zeng, Weidong Wu, Guobing Xu, Chi Xu, Bin Zheng, Yong Zhu, \\ Wei Zheng, Chun Chen \\ Department of Thoracic Surgery, Fujian Medical Union Hospital, Fuzhou, China \\ Correspondence to: Yong Zhu. Department of Thoracic Surgery, Fujian Medical Union Hospital, Fuzhou 350001, China. Email: 13509350188@163.com; \\ Bin Zheng. Department of Thoracic Surgery, Fujian Medical Union Hospital, Fuzhou 350001, China. Email: lacustrian@163.com.
}

\begin{abstract}
Lung cancer is the leading cause of cancer-related mortality worldwide. Patients with locally advanced non-small cell lung cancer (NSCLC) have lower overall survival. Studies have shown that some patients with unresectable stage III NSCLC develop disease progression after initial chemoradiotherapy, and new treatment is needed to improve the prognosis of these patients. The rapid development of therapy has greatly changed and continued to renew the treatment strategy of advanced NSCLC. However, the clinical treatment for patients with the wild-type gene remains problematic, and chemotherapy with platinum are not yet considered satisfactory. Herein, we are reporting a case of a patient with wild-type gene mutation locally advanced NSCLC who was treated with neoadjuvant therapy by using combined targeted anti-PD-1 immunotherapy and chemotherapy. The percentage of tumor cells with membranous PD-L1 staining (tumor proportion score) was $90 \%$ or greater. After receiving all three cycles of treatment, the patient underwent video-assisted right upper lung lobectomy and wedge resection plus radical mediastinal lymph node dissection. Pathological section samples showed a pathological complete response. This experience has led us to believe that the subgroup of patients with unresectable advanced NSCLC may benefit from this strategy and may have an opportunity for radical surgery.
\end{abstract}

Keywords: Immunotherapy; non-small cell lung cancer (NSCLC); pathological complete response

Submitted May 18, 2020. Accepted for publication Aug 21, 2020.

doi: $10.21037 /$ tlcr-20-770

View this article at: http://dx.doi.org/10.21037/tlcr-20-770

\section{Introduction}

Lung cancer is the leading cause of cancer-related mortality worldwide. Patients with locally advanced non-small cell lung cancer (NSCLC) have lower overall survival. For unresectable locally advanced NSCLC, radical concurrent chemoradiotherapy (CCRT) is recommended. Studies have shown that some patients with unresectable stage III NSCLC develop disease progression after initial chemoradiotherapy, and new treatment is needed to improve the prognosis of these patients. The optimal treatment of NSCLC patients who lack targetable mutations is still controversial (1). A platinum-based chemotherapy regimen remains the staple treatment for advanced wild-type gene NSCLC patients who lack targetable mutations. New drugs such as tyrosine kinase inhibitors (TKIs) and immunocheckpoint inhibitors have greatly changed the treatment strategy of advanced NSCLC and improved the prognosis of patients. The introduction of novel drugs and the use of new predictive biomarkers have resulted in a favorable prognosis for advanced NSCLC patients (2). Some clinical trials have focused attention on trying neoadjuvant therapy of combined immunotherapy with platinum-based chemotherapy for advanced NSCLC. Presently, the optimal therapeutic strategies for advanced NSCLC are still evolving. Some trials, such

\footnotetext{
$\wedge$ ORCID: 0000-0001-9692-8433.
} 


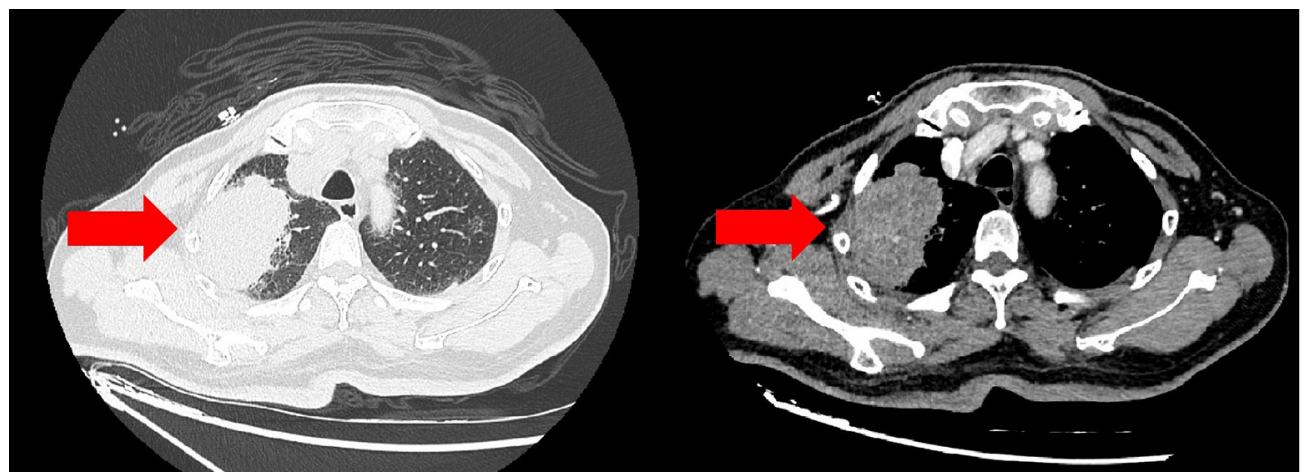

Figure 1 Chest computed tomography (CT) scan of the right upper lobe tumor before neoadjuvant therapy.

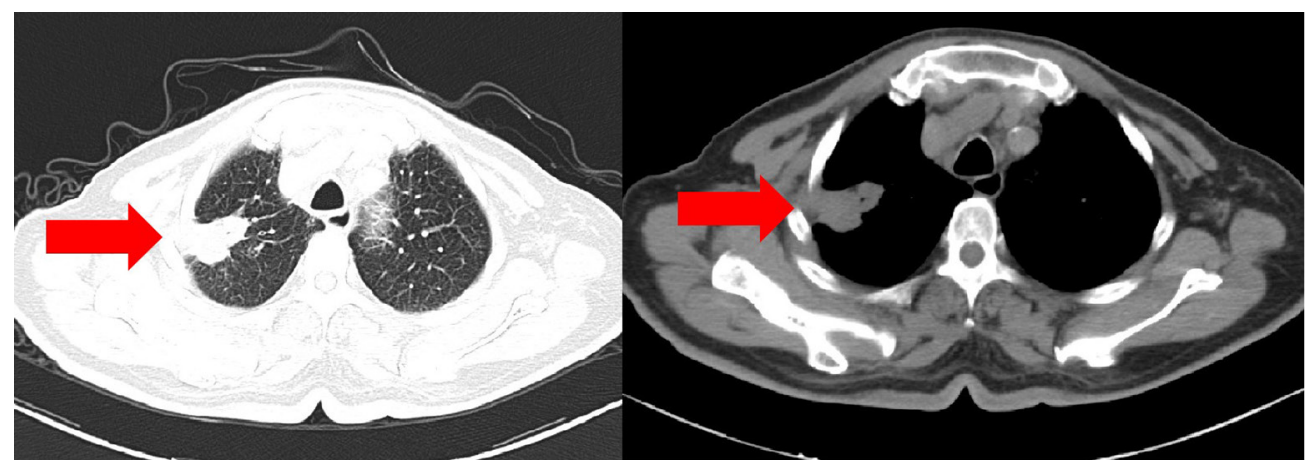

Figure 2 Chest computed tomography (CT) scan of the right upper lobe mass after neoadjuvant therapy.

as the KEYNOTE-189 (3) and IMPOWER-150 (4) trials have shown that immunotherapy combined with chemotherapy can bring more survival benefits to patients than chemotherapy alone. Herein, we are presenting a rare case of a patient with advanced NSCLC with the wildtype gene who achieved complete response to preoperative chemotherapy plus pembrolizumab.

We present the following case in accordance with the CARE reporting checklist (available at http://dx.doi. org/10.21037/tlcr-20-770).

\section{Case presentation}

A 77-year-old Chinese male smoker underwent chest computed tomography (CT) scanning which detected a pulmonary mass in the apical-posterior segment of the right upper lobe with a diameter of $65 \mathrm{~mm}$ (Figure 1). The patient did not complain of any related symptoms and no family history of carcinoma was found. Positronemission tomography/CT (PET/CT) showed an irregular hypermetabolic mass with lobulation in the right upper lobe, directly invading the pleura. It also showed an enlarged left hilar lymph node and mediastinal lymph node. Percutaneous lung puncture needle biopsy confirmed adenocarcinoma. Molecular testing of the pathological section sample was negative for epidermal growth factor receptor (EGFR), anaplastic lymphoma kinase (ALK), Kirsten rat sarcoma viral oncogene (KARS), rearranged during transfection (RET), and c-ros oncogene 1 receptor kinase (ROS1) mutations. The percentage of tumor cells with membranous PD-L1 staining (tumor proportion score) was $90 \%$ or greater. Given this, the patient's lung cancer was staged as cT3N2M0 (stage IIIB). There was no indication of radical surgical resection. Through careful multidisciplinary discussion, the patient was recommended three cycles of pemetrexed $\left(500 \mathrm{mg} / \mathrm{m}^{2}\right)$ and cisplatin $\left(75 \mathrm{mg} / \mathrm{m}^{2}\right)$, plus pembrolizumab $(200 \mathrm{mg})$. After receiving all three cycles of treatment, enhanced chest CT scan showed a partial response to this combination of neoadjuvant therapy (Figure 2), PET/CT also showed a partial response of abated metabolism and reduced volume of lung nodule and lymph node. Following multidisciplinary 

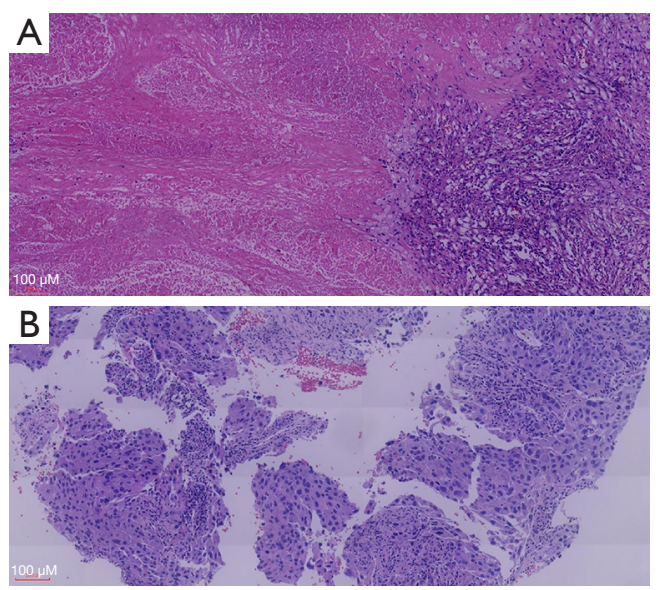

Figure 3 Pathological image. (A) Hematoxylin and eosin (HE) stain of the tumor bed (original magnification 100x) with no surviving tumor cells after neoadjuvant therapy. (B) HE stain of the tumor bed (original magnification 100x) with viable tumor cells before neoadjuvant therapy.

discussion, the patient underwent video-assisted right upper lung lobectomy and wedge resection plus radical mediastinal lymph node dissection. Pathological section samples revealed inflammatory cell infiltration, interstitial fibrosis with hyaline degeneration, cholesterol crystallization, and reactive hyperplasia of alveolar epithelium (Figure 3); no surviving tumor cells remained. Lymph nodes at levels 2, $2 \mathrm{~T}, 3 \mathrm{a}, 4,7,11,12$, and 13 were negative. And no adverse events occurred after therapy. The patient was followed up with duration of three months with confident, and there was no tumor recurrence.

All procedures performed in studies involving human participants were in accordance with the ethical standards of the institutional and/or national research committee(s) and with the Helsinki Declaration (as revised in 2013). Written informed consent was obtained from the patient for publication of this manuscript and any accompanying images.

\section{Discussion}

This case supports that neoadjuvant therapy of immunotherapy plus platinum-based chemotherapy can be an effective treatment for NSCLC. Though TKI therapy has the advantages of its great pertinence and limited side effects, but non-gene mutations and drug-resistance mutations render this targeted treatment ineffective on some patients. Meanwhile the high cost of targeted drugs also hinders its wider application. Nearly half of lung adenocarcinoma patients are negative for EGFR mutation (5), and almost 95\% of patients are negative for ALK mutation (6). The advent of immunotherapy has demonstrated a good curative effect for advanced NSCLC and provides hope for these patients. Indeed, a few new treatment strategies without targetable gene mutations for advanced NSCLC patients without targetable gene mutations have achieved survival benefits for advanced NSCLC patients. Neither chemotherapy nor immunotherapy alone as the neoadjuvant therapy for advanced NSCLC can be depended on to achieve meaningful pathological response (MPR). Langer et al. (7) reported that PD-1 inhibitor, combined with chemotherapy, could raise the objective response rate for patients with locally advanced NSCLC, confirming that the combination of immunotherapy and chemotherapy can work synergistically. Other clinical trials also showed that introducing PD-1 inhibitor as a first-line therapy could produce a good long-term curative effect $(3,8)$. The largest relative benefit was found in a group with a PD-L1 tumor proportion score of $50 \%$ or greater, which was consistent with the results of previous studies of PD-1 inhibition in advanced NSCLC (3,9-11). This experience has led us to believe that the subgroup of patients with unresectable advanced NSCLC may benefit from this strategy and may have an opportunity for radical surgery.

In conclusion, this case report showed an advanced NSCLC patient who achieved complete pathological response after short-term neoadjuvant combination therapy, although long-term follow-up is still needed to fully support the rationale of the curative strategy. We believe that immunotherapy combined with chemotherapy has the potential to overcome NSCLC and merits further research to gather better outcomes.

\section{Acknowledgments}

Funding: The study was supported by the Medical Innovation Project of Fujian Health and the Family Planning Commission (2015-CX-19), Fujian Provincial Major Special Projects (2017YZ0101), and Provincial Science and Technology Leading Project (2018Y0032).

\section{Footnote}

Reporting Checklist: The authors have completed the CARE reporting checklist. Available at http://dx.doi.org/10.21037/ tlcr-20-770 
Conflicts of Interest: All authors have completed the ICMJE uniform disclosure form (available at http://dx.doi. org/10.21037/tlcr-20-770). The authors have no conflicts of interest to declare.

Ethical Statement: The authors are accountable for all aspects of the work in ensuring that questions related to the accuracy or integrity of any part of the work are appropriately investigated and resolved. All procedures performed in studies involving human participants were in accordance with the ethical standards of the institutional and/or national research committee(s) and with the Helsinki Declaration (as revised in 2013). Written informed consent was obtained from the patient for publication of this manuscript and any accompanying images.

Open Access Statement: This is an Open Access article distributed in accordance with the Creative Commons Attribution-NonCommercial-NoDerivs 4.0 International License (CC BY-NC-ND 4.0), which permits the noncommercial replication and distribution of the article with the strict proviso that no changes or edits are made and the original work is properly cited (including links to both the formal publication through the relevant DOI and the license). See: https://creativecommons.org/licenses/by-nc-nd/4.0/.

\section{References}

1. Ettinger DS, Wood DE, Aisner DL, et al. Non-Small Cell Lung Cancer, Version 5.2017, NCCN Clinical Practice Guidelines in Oncology. J Natl Compr Canc Netw 2017;15:504-35.

2. Cohen AY, Kian W, Roisman LC, et al. Are we facing a cure in lung cancer?-KEYNOTE-001 insights. Ann Transl Med 2019;7:S215.

3. Chatwal MS, Tanvetyanon T. Combination chemotherapy and immunotherapy in metastatic non-small cell lung

Cite this article as: Gao P, Chen H, Zeng T, Wu W, Xu G, Xu C, Zheng B, Zhu Y, Zheng W, Chen C. A pathological complete response to neoadjuvant chemotherapy and immunotherapy in a non-small cell lung cancer patient. Transl Lung Cancer Res 2020;9(5):2157-2160. doi: 10.21037/tlcr-20-770 cancer: a setback for personalized medicine? Transl Lung Cancer Res 2018;7:S208-10.

4. Socinski MA, Jotte RM, Cappuzzo F, et al. Atezolizumab for First-Line Treatment of Metastatic Nonsquamous NSCLC. N Engl J Med 2018;378:2288-301.

5. Shi $\mathrm{Y}, \mathrm{Au} \mathrm{JS}$, Thongprasert $\mathrm{S}$, et al. A prospective, molecular epidemiology study of EGFR mutations in Asian patients with advanced non-small-cell lung cancer of adenocarcinoma histology (PIONEER). J Thorac Oncol 2014;9:154-62.

6. Wakuda K, Kenmotsu H, Serizawa M, et al. Molecular profiling of small cell lung cancer in a Japanese cohort. Lung Cancer 2014;84:139-44.

7. Langer CJ, Gadgeel SM, Borghaei H, et al. Carboplatin and pemetrexed with or without pembrolizumab for advanced, non-squamous non-small-cell lung cancer: a randomised, phase 2 cohort of the open-label KEYNOTE-021 study. Lancet Oncol 2016;17:1497-508.

8. Rizvi NA, Hellmann MD, Brahmer JR, et al. Nivolumab in Combination With Platinum-Based Doublet Chemotherapy for First-Line Treatment of Advanced Non-Small-Cell Lung Cancer. J Clin Oncol 2016;34:2969-79.

9. Garon EB, Rizvi NA, Hui R, et al. Pembrolizumab for the treatment of non-small-cell lung cancer. N Engl J Med 2015;372:2018-28.

10. Herbst RS, Baas P, Kim DW, et al. Pembrolizumab versus docetaxel for previously treated, PD-L1-positive, advanced non-small-cell lung cancer (KEYNOTE-010): a randomised controlled trial. Lancet 2016;387:1540-50.

11. Peters S, Gettinger S, Johnson ML, et al. Phase II Trial of Atezolizumab As First-Line or Subsequent Therapy for Patients With Programmed Death-Ligand 1-Selected Advanced Non-Small-Cell Lung Cancer (BIRCH). J Clin Oncol 2017;35:2781-9.

(English Language Editor: J. Gray) 